\section{Artículo por Invitación}

Pensar en Movimiento:

Revista de Ciencias del Ejercicio y la Salud ISSN 1659-4436

Vol. 14, No.2, pp. 22- 43

Cierre al 31 de diciembre, 2016

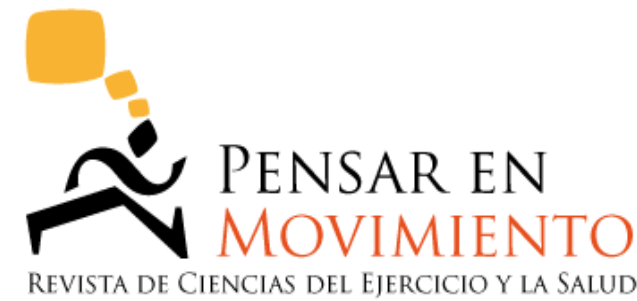

\title{
CONDUCTA OCUPACIONAL SEDENTARIA Y SOLUCIONES PARA AUMENTAR LA TERMOGÉNESIS NO ASOCIADA AL EJERCICIO (Versión traducida al español) ${ }^{1}$
}

\author{
Haley M. Scott, M.Sc. ${ }^{2(B, C, D, E)}$; Tess N. Tyton, M.Sc. ${ }^{2(B, C, D, E)}$; \\ Craig A. Horswill, Ph.D. 2(B,C,D,E) \\ horswill@uic.edu \\ 2Universidad de Illinois, Chicago, Illinois, \\ Estados Unidos de América \\ Envío original: 01/04/2016. Reenviado: 10/08/2016. \\ Aceptado: 05/09/2016. Publicado: 14/12/2016 \\ Doi: http://dx.doi.org/10.15517/pensarmov.v14i2.27496
}

\section{RESUMEN}

Scott, H.M., Tyton, T.N., \& Horswill, C.A. (2016). Conducta ocupacional sedentaria y soluciones para aumentar la termogénesis no asociada al ejercicio. PENSAR EN Movimiento: Revista de Ciencias del Ejercicio y la Salud, 14(2), 22-43. Conforme aumenta la prevalencia de la obesidad en todo el mundo, los investigadores buscan explicaciones para este fenómeno, en particular aquellas relevantes al gasto energético. Se ha identificado que la termogénesis no asociada al ejercicio, abreviada como NEAT (por sus siglas en inglés), es un componente discreto, pero a la vez considerable, del gasto energético diario total. Las exigencias de algunas ocupaciones limitan la disposición de tiempo para la actividad física planificada y claramente reducen la NEAT, lo cual contribuye a las conductas sedentarias que provocan la adiposidad aumentada. Se ha identificado específicamente al tiempo sentado prolongado durante la jornada laboral como un factor de riesgo para la obesidad y las enfermedades crónicas, independientemente de otros factores de riesgo existentes. Se han puesto en práctica algunas estrategias prácticas para aumentar la NEAT durante la jornada laboral: existen estaciones de trabajo que requieren mantener el equilibrio mientras se trabaja sentado en una pelota suiza (bola de ejercicio) o trabajar de pie, también otras en las que se pedalea sentado o se camina

\footnotetext{
${ }^{1}$ También disponible en la versión original en inglés en este mismo número.
} 
en una banda sin fin incorporada al escritorio, para contrarrestar los períodos prolongados de conducta sedentaria en el trabajo. Aunque hay pocos datos sobre los beneficios crónicos de estas estaciones, aquellas que promueven más movimiento - las estaciones de pedaleo o de caminata-aumentan los MET y el gasto energético total más que las otras alternativas. La desventaja de moverse más es que podría haber una disminución en la atención del trabajo de escritorio y, por lo tanto, una disminución en el funcionamiento cognitivo; sin embargo, los datos no son consistentes y puede ser que el beneficio para la salud sea igual o mayor que la desventaja de experimentar algunas pequeñas distracciones durante las tareas de escritorio.

Palabras claves: metabolismo; gasto energético; salud ocupacional.

La crisis mundial de obesidad observable hoy en las sociedades industrial y tecnológicamente desarrolladas puede explicarse por el exceso de energía ingerida, en comparación con la cantidad de energía que se gasta. La diferencia o balance entre el gasto energético diario total (GEDT), y la ingesta energética diaria, determina si un ser humano mantiene o cambia su peso corporal. Si bien es cierto que podría debatirse el papel de la eficiencia de almacenamiento y de gasto calórico como factores que aportan una contribución importante, las leyes de la termodinámica dictan que, si la ingesta de energía es mayor que el gasto, habrá un aumento de peso.

No está claro cuál lado de la ecuación del equilibrio energético ha cambiado más dramáticamente en las décadas recientes para llegar al apuro actual de salud. Sin embargo, muchos argumentarían que el estilo de vida sedentario es definitivamente uno de los principales factores. La advertencia "estar sentado es el nuevo fumado" expresa la preocupación (Mayo Clinic, 2014). Los avances de la tecnología y los objetivos del mundo de los negocios de aumentar constantemente la productividad, promueven la inactividad aún más, ya que casi todos los mecanismos para hacer negocios se pueden ejecutar por períodos prolongados de la jornada de trabajo sin que uno se aparte del escritorio.

Los objetivos de esta breve revisión son: a) definir y caracterizar los componentes de nuestro gasto energético diario; b) resumir el impacto del estilo de vida sedentario y el tiempo sentado prolongado, sobre los factores de riesgo de la obesidad y las enfermedades crónicas; c) identificar y describir el impacto de las estaciones de trabajo no tradicionales que podrían aumentar la termogénesis no asociada al ejercicio, conocida como NEAT por su abreviatura en inglés (non-exercise activity thermogenesis) y promover el movimiento a la vez que le permiten a la persona trabajadora el cumplimiento de las exigencias de su trabajo normal. Para este último objetivo, se resumirán los efectos agudos y crónicos de las estaciones de trabajo activas, así como los efectos de estas sobre la función cognitiva relevante a la productividad en las tareas de escritorio.

Las publicaciones científicas que respaldan esta revisión se obtuvieron de búsquedas en PubMed y Google Scholar. Las palabras clave y las frases fueron todas en inglés, e incluyeron: gasto calórico (calorie expenditure), cognitivo (cognitive), escritorio (desk), gasto energético (energy-expenditure), metodología para gasto energético (energy-expenditure methodology), 
tembladera (fidgeting), salud (health), factores de riesgo para la salud (health risk factors), inactividad (inactivity), termogénesis por actividad que no es ejercicio (non-exercise activity thermogenesis), tiempo sentado en jornada laboral (occupational sitting), consumo de oxígeno (oxygen consumption), estación de trabajo con pedales (pedal workstation), inactividad física (physical inactivity), tasa metabólica en reposo (resting metabolic rate), factores de riesgo (risk factors), tasa metabólica en posición sentada (seated metabolic rate), sedentario (sedentary), conducta sedentaria (sedentary behavior), sentarse (sitting), tasa metabólica en posición sentada (sitting metabolic rate), estación de trabajo con pelota suiza (stability ball work station), estar de pie (standing), estación para trabajar de pie (standing workstation), escritorio para trabajar de pie (standing desk), efecto termogénico de los alimentos (thermogenic effect of food), gasto energético diario total (total daily energy expenditure), estación de trabajo (workstation), estación de trabajo con banda sin fin (workstation treadmill). Para las secciones sobre tiempo sentado prolongado y estaciones de trabajo, la búsqueda de información se hizo a partir del año 2000.

\section{Gasto energético diario total}

La energía que gastan los seres humanos en un período de 24 horas define el GEDT. La Figura 1 presenta los componentes del GEDT como tres procesos distintos que gastan calorías: el metabolismo basal, el efecto termogénico de los alimentos (ETA) y la actividad física (Levine, 2005). La tasa metabólica basal (TMB) es la energía utilizada para la homeostasis de las células, los tejidos y los órganos en reposo. La TMB representa la proporción más grande del GEDT, entre 60 y 75\%, y depende principalmente de la masa del tejido con mayor actividad metabólica, el tejido magro (Hill, Melby, Johnson, \& Peters, 1995). Por lo tanto, la TMB se ve influenciada por la edad, la estatura, la masa corporal y el sexo; también se puede calcular tomando en cuenta estos factores. El ETA es el costo obligatorio propio de la digestión y metabolismo de los alimentos, con la menor contribución de aproximadamente $10 \%$ del GEDT. Este costo pareciera ser bastante estable, modificable únicamente mediante la manipulación de la composición de la dieta (Hill et al., 1995), pero aún esa manipulación tendría relativamente poco impacto sobre el GEDT (Hill et al., 1995). La actividad física representa el 15 o 30\% restante. Es el proceso más variable, y puede ser el más dependiente de nuestro comportamiento, comparado con la resistencia al cambio mostrada por la TMB y el ETA.

Para la discusión del GEDT y el estilo de vida sedentario, el componente de actividad física se ha dividido en dos: la actividad planificada, es decir, el ejercicio, y el NEAT, que se puede considerar como aquel movimiento que no alcanza el umbral para mejorar la aptitud física pero que, desde un punto de vista acumulativo, representa una cantidad apreciable de calorías durante el día. La actividad espontánea se considera parte del NEAT. Los primeros trabajos para intentar la separación entre el ejercicio y el NEAT indicaron que la tembladera, una forma de NEAT, podría aportar un gasto de hasta $3350 \mathrm{~kJ}$ por día $(800 \mathrm{kcal} / \mathrm{d}$ ) (Ravussin, Lillioja, Anderson, Christin, \& Bogardus, 1986). Mediante la utilización de un cuarto para

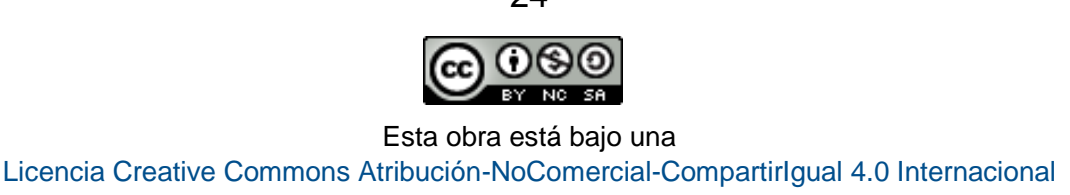


calorimetría y detectores de movimiento, Ravussin y sus colegas fueron capaces de seccionar los componentes del gasto energético diario total en 177 sujetos que permanecieron en la cámara durante $24 \mathrm{~h}$ (Ravussin et al., 1986). Ellos le atribuyeron al movimiento espontáneo un promedio de $8.7 \%$ (rango = 3.9 a $16.6 \%$ ) del GEDT, conforme a las mediciones de consumo de oxígeno y valoración del movimiento. El efecto acumulado del NEAT a lo largo de los años podría explicar por qué algunos individuos se mantienen relativamente magros y con una masa corporal normal sin involucrarse formalmente en el ejercicio o sin restringir su ingesta calórica (Johannsen \& Ravussin, 2008; Ravussin et al., 1986; Levine, 2002).

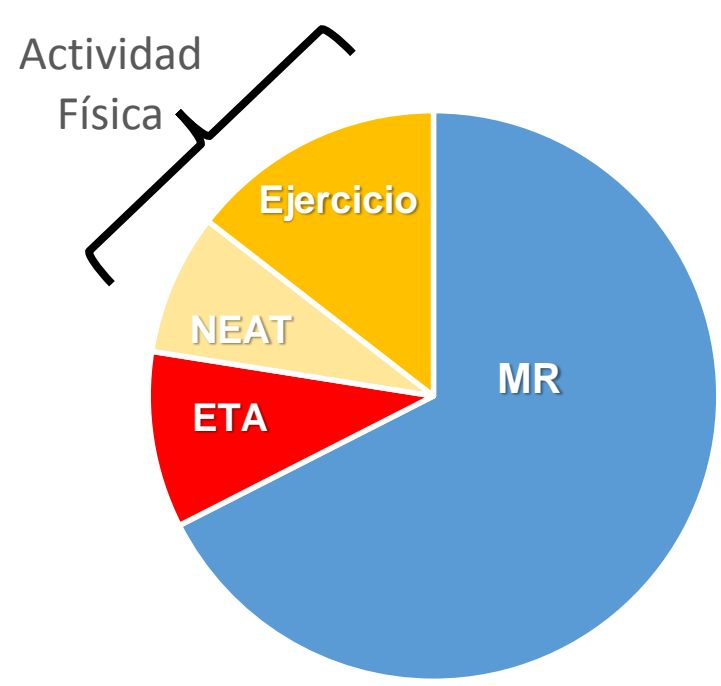

Figura 1. Componentes del gasto energético diario total y sus respectivas proporciones aproximadas. MR: metabolismo en reposo. ETA: efecto termogénico de los alimentos. NEAT: termogénesis no asociada al ejercicio.

Los componentes del GEDT están relacionados entre sí, de manera que el cambio en un componente puede afectar a otro. Hill et al. (1995) describieron los efectos teóricos del ejercicio planificado sobre la masa corporal: si no ocurre una compensación (esto es, si las personas que empiezan a hacer ejercicio no ingieren más energía), habrá una reducción inicial de la masa grasa. Posteriormente, una vez que el cuerpo alcance el punto de asentamiento descrito por los mismos autores, el déficit energético continuado debido al ejercicio causará una pérdida de masa libre de grasa, la cual a su vez reduciría la TMB y el GEDT. En la práctica, esta relación podría no funcionar como se esperaría y podría verse complicada por otros factores como la raza (DeLany, et al., 2014). La disminución en la ingesta energética, esto es, "hacer dieta”, produciría una disminución en el ETA y también podría provocar una disminución en la 
actividad física diaria y en el gasto energético diario total (Redman et al., 2009). Por otra parte, Blaak, Westerterp, Bar-Or, Wouters, \& Saris (1992) y Racette, Schoeller, Kushner, \& Neil (1995) mostraron que las personas obesas sometidas a un programa de ejercicio planificado aumentan la actividad espontánea y posiblemente también el GEDT, pues este último aumentó más allá de lo que se predijo por añadir el ejercicio formal.

Los resultados de otros estudios son un poco contradictorios: algunos muestran que no ocurre una disminución en la actividad espontánea ni en el gasto energético como resultado de la participación en un programa de ejercicio (Willis, et al., 2014), mientras otros registran una aparente disminución, ya que no hay reducción del peso (Thivel, Chaput, Adamo, \& Goldfield, 2014). El efecto de un programa de ejercicio planificado sobre el gasto energético espontáneo podría depender de la población bajo estudio, es decir, magros vs. obesos o mayores vs. jóvenes. Además, el tipo de programa de ejercicios, a saber, si es entrenamiento aeróbico o contra resistencia, podría tener un efecto distinto sobre el gasto energético por actividad espontánea (Drenowatz, Grieve, \& DeMello, 2015). Actualmente, se desconoce si el inverso es cierto, a saber, que un aumento en las actividades que aumentan el NEAT podrían además incrementar la participación del individuo en el ejercicio planificado.

Independientemente de lo anterior, la contribución del NEAT al GEDT es lo suficientemente fascinante como para incitar a explorar las opciones que produzcan un aumento del NEAT. Estas opciones se están ofreciendo y estudiando en el contexto laboral, ya que este contexto ha sido un factor muy importante en el aumento de la inactividad; la última sección de esta revisión presenta un resumen de estas investigaciones. A lo largo de las últimas dos décadas, la falta de actividad física diaria, o su disminución, es una preocupación creciente por su relación con la salud y la mortalidad, dando como resultado la unificación de la comunidad médica para recomendar criterios para el ejercicio y la salud (Pate et al., 1995; Haskell et al., 2007).

\section{El tiempo sentado prolongado y sus riesgos para la salud}

El tiempo sentado como conducta sedentaria. Los expertos se mantienen en un debate constante sobre la definición más correcta de conducta sedentaria, de modo que se distinga de inactividad física, a saber, cuando no se alcanza el volumen recomendado de ejercicio semanal para obtener beneficios de aptitud física y salud. Las actividades sedentarias son aquellas que se dan durante la vigilia y provocan una tasa metabólica de 1.0 a 1.5 en equivalentes metabólicos (METS), según el Compendio de Actividades Físicas (Ainsworth et al., 2000; Pate, O'Neill, \& Lobelo, 2008). La Red para la Investigación sobre la Conducta Sedentaria (Sedentary Behaviour Research Network, 2012) añadió el detalle de posición a la definición para ayudar a especificar el término: se trata de una conducta que genera un metabolismo $\leq 1.5$ METS en una posición sentada o reclinada. El debate se ve complicado por la observación con respecto a que se pueden obtener beneficios para la salud mediante conductas que no superan el límite de METS en ninguna de estas dos definiciones, pero al menos superan la categoría de estar sentado, a saber, estar de pie (Gibbs, Hergenroeder,

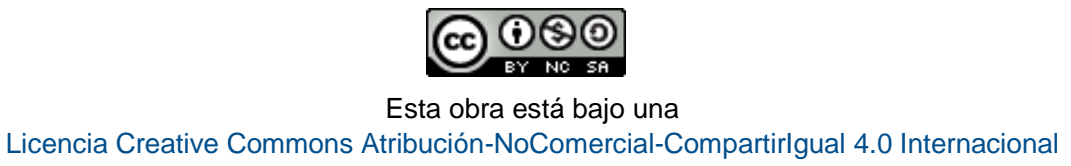


Katzmarzyk, Lee, \& Jakicic, 2015). Para efectos de esta revisión, se considera conducta sedentaria estar sentado frente a un escritorio por tiempo prolongado con movimientos mínimos.

Durante las horas de vigilia, la persona promedio gasta más de la mitad del día involucrada en conductas sedentarias (Matthews et al., 2008), y estar sentado es una de las formas más comunes. Estar sentado ocurre mientras viajamos, durante la mayoría de las reuniones, mientras se practican actividades de ocio específicas como ver televisión y jugar juegos de computadora, y en la estación de trabajo. Según indican algunos estudios epidemiológicos, el tiempo que se pasa sentado aumenta con la edad (Matthews et al., 2008). $\mathrm{Si}$ bien es cierto que se puede disminuir el tiempo de conductas sedentarias mediante la participación en actividades recreativas que exigen movimiento, para muchas personas no es factible disminuir el tiempo sentadas debido a las obligaciones de su trabajo.

El tiempo sentado durante la jornada laboral (pasar sentado por el trabajo). En algunas profesiones como la administración, el transporte y la tecnología, los empleados deben pasar la mayor parte de su jornada laboral frente a un escritorio o el volante, con una cantidad mínima de movimiento. Se ha acuñado la expresión "pasar sentado por el trabajo" (occupational sitting) para referirse a esta conducta inactiva. En algunos estudios sobre las conductas de los trabajadores se pueden ver ejemplos de la magnitud del problema: los empleados de un centro de llamadas telefónicas pasaron un promedio de $6.6 \mathrm{~h}$ de su jornada laboral frente al escritorio, mientras que otro estudio encontró una duración similar de $6.2 \mathrm{~h}$ sentados en el trabajo (Ryde et al., 2014; Thorp et al., 2012). La preocupación va más allá: para los hombres, un ambiente sedentario en el trabajo promueve la participación en actividades recreativas físicamente inactivas fuera de la jornada laboral (Gimeno et al., 2009). La combinación del sedentarismo en el trabajo con el sedentarismo durante el tiempo de ocio va sumando a la inactividad física general día tras día. Al mantener un bajo gasto energético durante la mayor parte del día se promueven el sobrepeso y la obesidad, además de un riesgo aumentado de enfermedades crónicas a lo largo del tiempo (ver la Tabla 1).

Riesgos para la salud. Aunque es generalmente aceptado que la inactividad física tiene consecuencias negativas para varios riesgos de la salud y para la mortalidad por múltiples causas, cada vez se publican más investigaciones centradas en los riesgos para la salud relacionados con pasar mucho tiempo sentado durante el trabajo, independientemente de la inactividad general. La inactividad física general tal como la ausencia de ejercicio regular, está claramente asociada con el desarrollo de la obesidad, la enfermedad de las arterias coronarias, la diabetes tipo 2, varios tipos de cáncer, y la mortalidad prematura (Lee et al., 2012). Aquellas personas que trabajan en profesiones en las cuales deben pasar sentados la mayor parte de las horas laborales podrían estarle haciendo un daño aún mayor a su cuerpo de lo que se imaginan: contrario a la suposición de que el ejercicio regular al inicio o al final del día es suficiente para deshacer el daño de la inactividad física diaria, al menos un estudio sugiere que 
Tabla 1

Resumen de publicaciones sobre riesgos para la salud asociados a pasar tiempo sentado

\begin{tabular}{|c|c|c|c|c|c|c|}
\hline $\begin{array}{l}\text { Riesgo } \\
\text { para la } \\
\text { salud }\end{array}$ & Estudio & Diseño & Muestra & Variable & $\begin{array}{c}\text { Tiempo } \\
\text { sentado por } \\
\text { trabajo }\end{array}$ & Resultados \\
\hline \multirow{3}{*}{$\begin{array}{l}\text { Sobrepeso } \\
\text { / Obesidad }\end{array}$} & $\begin{array}{l}\text { Mummery } \\
\text { et al., } 2005\end{array}$ & $\begin{array}{l}\text { Corte } \\
\text { transversal }\end{array}$ & $\begin{array}{l}n= \\
1,579\end{array}$ & $\mathrm{IMC} \geq 25$ & $>6$ horas $/ d$ & $\begin{array}{l}\text { Asociación entre sobrepeso } \\
\text { y obesidad, y el tiempo } \\
\text { sentado por el trabajo en } \\
\text { hombres }\end{array}$ \\
\hline & $\begin{array}{l}\text { Lin et al., } \\
2015\end{array}$ & $\begin{array}{l}\text { Longitudinal } \\
\text { de } 8 \text { años }\end{array}$ & $\begin{array}{l}n= \\
5,285\end{array}$ & IMC & Media $=3 \mathrm{~h} / \mathrm{d}$ & $\begin{array}{l}\text { Relación directa significativa } \\
\text { entre el tiempo sentado y } \\
\text { aumento en IMC }\end{array}$ \\
\hline & $\begin{array}{l}\text { Eriksen et } \\
\text { al., } 2015\end{array}$ & $\begin{array}{l}\text { Longitudinal } \\
\text { de } 5 \text { años }\end{array}$ & $\begin{array}{l}n= \\
4,732\end{array}$ & IMC & $\begin{array}{l}\text { Media: } \geq 25 \\
\text { h/semana }\end{array}$ & $\begin{array}{l}\text { Asociación entre tiempo } \\
\text { sentado en el trabajo y } \\
\text { aumento en IMC en mujeres }\end{array}$ \\
\hline \multirow{3}{*}{$\begin{array}{l}\text { Enferme- } \\
\quad \text { dad } \\
\text { Cardio- } \\
\text { vascular }\end{array}$} & $\begin{array}{l}\text { Katzmarzyk } \\
\text { et al., } 2009\end{array}$ & $\begin{array}{l}\text { Longitudinal } \\
\text { de } 12 \text { años }\end{array}$ & $\begin{array}{l}n= \\
17,013\end{array}$ & $\begin{array}{l}\text { Muerte por } \\
\text { enf. CV }\end{array}$ & $\begin{array}{l}\text { Tiempo diario } \\
\text { sentado total }\end{array}$ & $\begin{array}{l}\text { Dosis/respuesta entre } \\
\text { tiempo sen-tado y muerte } \\
\text { por enfermedad CV }\end{array}$ \\
\hline & $\begin{array}{l}\text { Warren et } \\
\text { al., } 2010\end{array}$ & $\begin{array}{l}\text { Longitudinal } \\
\text { de } 21 \text { años }\end{array}$ & $\begin{array}{l}n= \\
7,744\end{array}$ & $\begin{array}{l}\text { Muerte por } \\
\text { enfermedad } \\
\text { CV }\end{array}$ & $\begin{array}{l}23 \mathrm{~h} / \text { semana } \\
\text { viajando } \\
\text { sentado o } \\
\text { viendo TV }\end{array}$ & $\begin{array}{l}\text { Riesgo de muerte por } \\
\text { enfermedad CV aumentado } \\
\text { en } 64 \%\end{array}$ \\
\hline & $\begin{array}{l}\text { Altieri et } \\
\text { al., } 2004\end{array}$ & $\begin{array}{l}\text { Casos y } \\
\text { controles, } 4 \\
\text { años }\end{array}$ & $n=507$ & $\begin{array}{l}\text { Incidencia de } \\
\text { infarto agudo } \\
\text { al miocardio }\end{array}$ & $\begin{array}{l}\text { Nivel de } \\
\text { actividad física } \\
\text { en el trabajo }\end{array}$ & $\begin{array}{l}\text { Relación inversa entre } \\
\text { tiempo sentado e incidencia } \\
\text { de infarto agudo al miocardio }\end{array}$ \\
\hline \multirow[b]{2}{*}{$\begin{array}{l}\text { Disfunción } \\
\text { Metabólica }\end{array}$} & $\begin{array}{l}\text { Hu et al., } \\
2003\end{array}$ & $\begin{array}{l}\text { Longitudinal } \\
\text { de } 12 \text { años }\end{array}$ & $\begin{array}{l}n= \\
14,290\end{array}$ & $\begin{array}{l}\text { Incidencia } \\
\text { de diabetes } \\
\text { tipo } 2\end{array}$ & $\begin{array}{l}\text { Actividad física } \\
\text { liviana en el } \\
\text { trabajo }\end{array}$ & $\begin{array}{l}\text { Incidence of type } 2 \text { diabetes } \\
\text { was highest in those with } \\
\text { lowest levels of occupational } \\
\text { physical activity }\end{array}$ \\
\hline & $\begin{array}{l}\text { Chu \& } \\
\text { Moy, } 2013\end{array}$ & $\begin{array}{l}\text { Corte } \\
\text { transversal }\end{array}$ & $n=686$ & $\begin{array}{l}\text { Incidencia de } \\
\text { factores } \\
\text { relacionados } \\
\text { con } \\
\text { enfermedad } \\
\text { metabólica }\end{array}$ & $\begin{array}{l}\text { Bajos niveles de } \\
\text { actividad física } \\
\text { por el trabajo, } \\
\text { trans-porte y } \\
\text { tareas del hogar }\end{array}$ & $\begin{array}{l}\text { Los niveles bajos de } \\
\text { actividad física en todas las } \\
\text { áreas se asocian con mayor } \\
\text { riesgo de enfermedad } \\
\text { metabólica }\end{array}$ \\
\hline \multirow{3}{*}{ Cáncer } & $\begin{array}{l}\text { Thune et } \\
\text { al., } 1997\end{array}$ & $\begin{array}{l}\text { Longitudinal } \\
\text {-de } 13 \text { años }\end{array}$ & $\begin{array}{l}n= \\
25,624\end{array}$ & $\begin{array}{l}\text { Incidencia } \\
\text { de cáncer de } \\
\text { mama }\end{array}$ & $\begin{array}{l}\text { Nivel de act. } \\
\text { física en el } \\
\text { trabajo (seden- } \\
\text { tario a trabajo } \\
\text { manual) }\end{array}$ & $\begin{array}{l}\text { Los niveles más altos de } \\
\text { actividad física en el trabajo } \\
\text { se asocian con menor riesgo } \\
\text { de cáncer de mama }\end{array}$ \\
\hline & $\begin{array}{l}\text { Levi et al., } \\
1999\end{array}$ & $\begin{array}{l}\text { Casos y } \\
\text { controles de } 5 \\
\text { años }\end{array}$ & $n=620$ & $\begin{array}{l}\text { Riesgo de } \\
\text { cáncer de } \\
\text { mama }\end{array}$ & $\begin{array}{l}\text { Tiempo senta- } \\
\text { do por trabajo } \\
\text { ("más que nada } \\
\text { sentado") }\end{array}$ & $\begin{array}{l}\text { El riesgo más alto de cáncer } \\
\text { de mama se asocia con el } \\
\text { nivel más alto de pasar } \\
\text { sentado por el trabajo }\end{array}$ \\
\hline & $\begin{array}{l}\text { Tavani et } \\
\text { al., } 1999\end{array}$ & $\begin{array}{l}\text { Casos y } \\
\text { controles, } 5 \\
\text { años }\end{array}$ & $\begin{array}{l}n= \\
5,379\end{array}$ & $\begin{array}{l}\text { Riesgo de } \\
\text { cáncer rectal } \\
\text { o del colon }\end{array}$ & $\begin{array}{l}\text { Tiempo senta- } \\
\text { do por trabajo } \\
\text { ("más que nada } \\
\text { sentado") }\end{array}$ & $\begin{array}{l}\text { La actividad física en la } \\
\text { jornada laboral protege del } \\
\text { cáncer de colon }\end{array}$ \\
\hline
\end{tabular}

Fuente: elaboración propia 
esta estrategia no compensa los efectos nocivos de la conducta sedentaria prolongada, entre la cual está pasar sentado por el trabajo (Matthews et al., 2012). Más aún, en un estudio que mostró que un $7 \%$ de las muertes se debieron a pasar demasiado tiempo sentados, no hubo una disminución en el riesgo al realizar 30 minutos de ejercicio moderado por día (la recomendación del $\mathrm{ACSM}$ ), asimismo, el cociente de riesgo fue $\geq 1$ si una persona pasaba sentada entre 8 y 11 horas al día (Van der Ploeg, Chey, Korda, Banks, \& Bauman, 2012).

Entre los riesgos para la salud que presenta pasar sentado por el trabajo están el sobrepeso y la obesidad. En la Tabla 1 se presenta un resumen de varios estudios de gran escala que respaldan esta afirmación. El trabajo de Lin, Courtney, Lombardi, \& Verma, (2015) y Eriksen, Rosthøj, Burr, \& Holtermann (2015) muestra cómo el desarrollo de la obesidad está relacionado con las horas que la gente pasa sentada, de una manera que sugiere un grado de causa-efecto que va más allá de una simple correlación. Los períodos prolongados de gasto calórico mínimo, como pasar sentada, aumentan la probabilidad de un superávit energético y el comienzo de la obesidad en la persona. Por ejemplo, el estudio realizado por Eriksen y sus colegas (2015) reveló que cuando las mujeres pasaron sentadas un promedio de 25 h o más por semana, se dio un aumento del IMC en un período de 5 años. La combinación del sobrepeso o la obesidad con pasar largos ratos del día sentadas casi sin moverse complica los riesgos para la salud de las personas.

El sobrepeso y la obesidad aumentan el riesgo de desarrollar enfermedades cardiovasculares. No está totalmente claro que exista una relación directa entre pasar sentado por el trabajo y la aparición de enfermedades cardiovasculares, independiente de la adiposidad; sin embargo, la correlación sí se ha identificado (Altieri, Tavani, Gallus, \& La vecchia, 2004; Warren et al, 2010). El riesgo de desarrollar una enfermedad cardiovascular o de sufrir de alguna afección relacionada se incrementa conforme hay un exceso de tiempo sentado (Tabla 1). Un estudio realizado por Altieri y sus colegas (2004) mostró que quienes pasaron más tiempo sentados por el trabajo aumentaron su riesgo de tener un infarto agudo al miocardio en un lapso de 4 años. Estos investigadores encontraron que 11\% de los infartos agudos al miocardio se pueden explicar por pasar un tiempo prolongado sentados en el trabajo.

La disfunción metabólica, que incluye el síndrome metabólico, es otro resultado del almacenamiento excesivo de energía, la adiposidad y la inactividad. La resistencia a la insulina, la glucosa sanguínea elevada, el colesterol alto y la hipertensión son componentes de la disfunción y anteceden a la diabetes tipo 2 y la enfermedad cardiovascular. Se ha asociado el pasar demasiado tiempo sentado, inclusive el pasar tiempo sentado por trabajo, con la disfunción metabólica. Un estudio longitudinal de 12 años realizado en Finlandia encontró que la incidencia de diabetes tipo 2 es mucho más baja en aquellas personas que son físicamente más activas en el trabajo en comparación con aquellas que son mayormente sedentarias (Hu et al., 2003). Esta asociación fue evidente luego de hacer ajustes por la conducta sedentaria durante el traslado hacia el trabajo y desde este, así como durante el tiempo de ocio. Si bien no está claro si se trata de una relación de causalidad, los resultados de este estudio señalan la necesidad de disminuir el tiempo sedentario durante el trabajo. 
Recientemente, se ha examinado el efecto de pasar sentado sobre el desarrollo de distintos tipos de cáncer. A pesar de que los datos son relativamente escasos, se ha establecido una asociación entre pasar sentado por el trabajo por tiempo prolongado y el cáncer de mama y de colon. Un estudio longitudinal de 13 años de duración realizado por Thune, Brenn, Lund, \& Gaard (1997) reveló que los niveles más altos de actividad física en el trabajo están relacionados con un menor riesgo de cáncer de mama. Levi, Pasche, Lucchini, \& La Vecchia (1999) encontraron que los volúmenes mayores de tiempo sedentario por trabajo estaban asociados con el riesgo más alto de desarrollar cáncer de mama entre las mujeres de su muestra. El riesgo de cáncer del colon también disminuyó conforme aumentaron los niveles de actividad física en el trabajo, aunque no fue así con el cáncer rectal (Tavani et al., 1999). En términos generales, pareciera que los largos períodos de conducta sedentaria son un peligro para la salud y un catalizador para la obesidad y las enfermedades metabólicas.

\section{Estaciones de trabajo}

Debido a que muchas personas tienen trabajo de escritorio y pasan potencialmente dos terceras partes del día sentadas, hay una necesidad apremiante de incorporar la actividad física no asociada al ejercicio o algún tipo de actividad física planificada a sus vidas (Ryde et al., 2014). Al considerar algunas alternativas a la estación de trabajo típica en la cual se trabaja sentado, con el propósito de aumentar el gasto energético y el movimiento durante la jornada laboral, es importante que la actividad no interfiera con la productividad. En aquellas situaciones en las cuales es necesario pasar mucho tiempo sentado para alcanzar los objetivos del trabajo, podría ser beneficioso interrumpir los extensos ratos de trabajo sedentario con breves períodos para caminar o ejecutar distintos movimientos o actividades intermitentes.

Actualmente, existen cuatro estaciones de trabajo distintas para promover el gasto energético y el movimiento, a saber: 1) un balón suizo como asiento, 2) un escritorio para trabajar de pie o ajustable para alternar entre trabajo de pie y sentado, 3) un dispositivo con pedales incorporado debajo del escritorio y 4) un escritorio con banda sin fin para caminar. Invitamos al lector a consultar el trabajo de Tudor-Locke, Schuna, Frensham, \& Proenca (2014), en el cual se presenta un resumen exhaustivo de la terminología y los aspectos críticos de las estaciones de trabajo. Mientras tanto, para efectos de esta revisión, clasificaremos el sentarse en un balón suizo y el trabajar de pie como estrategias reactivas o estáticas: los movimientos que ocurren obedecen principalmente a pequeños desplazamientos del centro de gravedad o ajustes posturales en una misma posición. En términos generales, las estaciones de trabajo reactivas son muy prácticas y están ampliamente disponibles. Sin embargo, podría ser que el movimiento generado no sea suficiente como para elevar el gasto energético o producir beneficios para la salud. Por otra parte, las estaciones de trabajo con pedales o con bandas sin fin las clasificaremos como proactivas o dinámicas. Estas alternativas facilitan la ejecución de movimientos rítmicos a intensidad variable. Si bien es cierto que las estaciones de trabajo proactivas provocan más movimiento y un mayor gasto energético, su costo y sentido

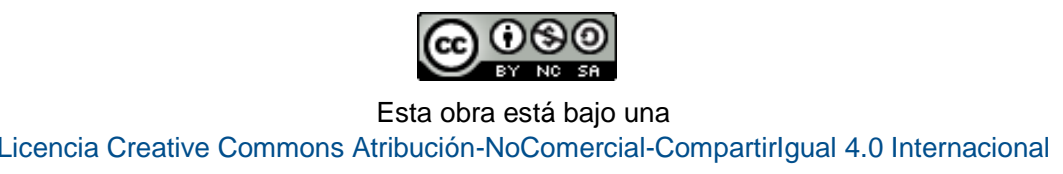


práctico pueden ser factores limitantes. Además, debido a que el esfuerzo físico es intencional, la atención mental necesaria podría disminuir la productividad en el trabajo. El resto de esta revisión se dedicará a lo que muestran las investigaciones acerca de los beneficios agudos y crónicos de la utilización de estos cuatro tipos de estaciones de trabajo, así como su efecto sobre el rendimiento cognitivo.

Respuestas agudas al uso de estaciones de trabajo alternativas. Mediante el aumento del movimiento durante la utilización de estaciones de trabajo alternativas, se esperaría que ocurrieran respuestas fisiológicas agudas en términos de gasto energético, frecuencia cardíaca y presión arterial. Las respuestas agudas son fáciles de medir y podrían explicar por qué existen más investigaciones para estos efectos inmediatos que para los efectos crónicos. A pesar de ser pasajeras, las respuestas agudas diarias podrían tener un efecto acumulado que posiblemente contribuya a los cambios crónicos para así modificar el riesgo de enfermedad.

Gasto energético. El aumento en el gasto energético diario debido al uso de estaciones de trabajo alternativas ayuda a compensar la ingesta calórica de cada día, de modo que pueden ocurrir efectos beneficiosos para cada persona en el largo plazo. La evidencia indica que la tasa de gasto energético varía dependiendo de la categoría de estación de trabajo utilizada. Esto se puede observar en la Figura 2, en la cual se resumen los valores promedio de gasto energético publicados para diversas estaciones de trabajo. El patrón sugiere que, en términos generales, si bien es cierto que las estaciones de trabajo reactivas elevan el metabolismo, el gasto se mantiene más bajo que el de las estaciones de trabajo proactivas. En un caso, el gasto energético durante el uso de una estación reactiva no se elevó por encima del obtenido al estar simplemente sentado en una estación de trabajo convencional (Speck y Schmitz 2011).

Un examen más profundo de la utilización del balón suizo para sentarse muestra que pueden ocurrir ligeros aumentos en el gasto energético, debidos a la superficie cambiante y a los movimientos reactivos para mantener el equilibrio. Por ejemplo, Dickin, Surowiec, \& Wang (2015) midieron el gasto energético de los trabajadores en una silla plana, en una silla acolchada o en un balón suizo. Se calculó el gasto energético promedio según el consumo de oxígeno y la producción de dióxido de carbono durante tres tareas de diez minutos cada una. Los resultados mostraron una diferencia en el gasto energético al comparar la silla plana $(6.1 \pm$ $1.0 \mathrm{~kJ} / \mathrm{min}$ o $1.46 \pm 0.25 \mathrm{kcal} / \mathrm{min})$ con el balón suizo $(6.9 \pm 0.8 \mathrm{~kJ} / \mathrm{min} 01.65 \pm 0.20 \mathrm{kcal} / \mathrm{min})$. La utilización del balón suizo y la silla acolchada promovió un gasto energético mayor en comparación con la silla plana: $10.4 \%(p=0.01)$ mayor en el balón suizo y $9.6 \%(p=0.03)$ mayor en la silla acolchada, lo cual sugiere que la superficie inestable podría ser una manera efectiva de forzar la contracción muscular. Además, algunos estudios sobre los escritorios para trabajar de pie cuestionan su valor, específicamente en lo que respecta al gasto energético, puesto que los resultados que se han reportado son de pequeños aumentos o de los que no son estadísticamente significativos, ni en niños (Benden, Zhao, Jeffrey, Wendel, \& Blake,

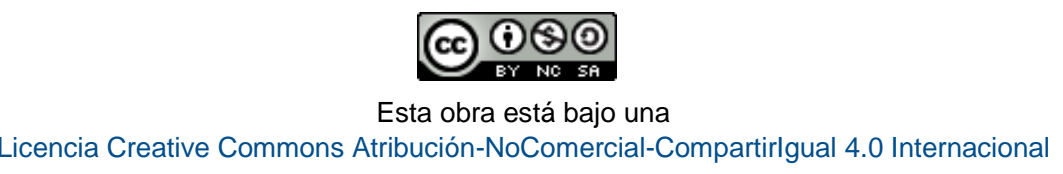


2014), ni en adultos (Reiff, Marlatt, \& Dengel, 2012; Speck and Schmitz, 2011). No obstante, tanto Benden et al. (2014) como Reiff et al. (2012) explicaron la utilidad de poner en práctica el uso de escritorios para trabajar de pie en las aulas: a pesar de que los aumentos en el gasto energético son relativamente pequeños, la actividad leve que hacen los niños y los estudiantes de edad universitaria podría ser beneficiosa en el largo plazo por la acumulación del efecto a lo largo de toda una vida. Por el contrario, Speck y Schmitz (2011) no reportaron diferencias en el metabolismo durante el trabajo en computadora con la utilización de estaciones de trabajo alternativas (balón suizo o de pie) en adultos inactivos que en su mayoría tenían sobrepeso (promedio de IMC = 27) y cuestionaron que dichas estaciones pudieran contribuir al NEAT, con los beneficios concomitantes para la salud, en todas las personas.

$\bullet A \odot B \odot C \odot D \bullet E \odot F \bullet G \odot H \bullet I \bullet J$

\section{GE, kcal/min}

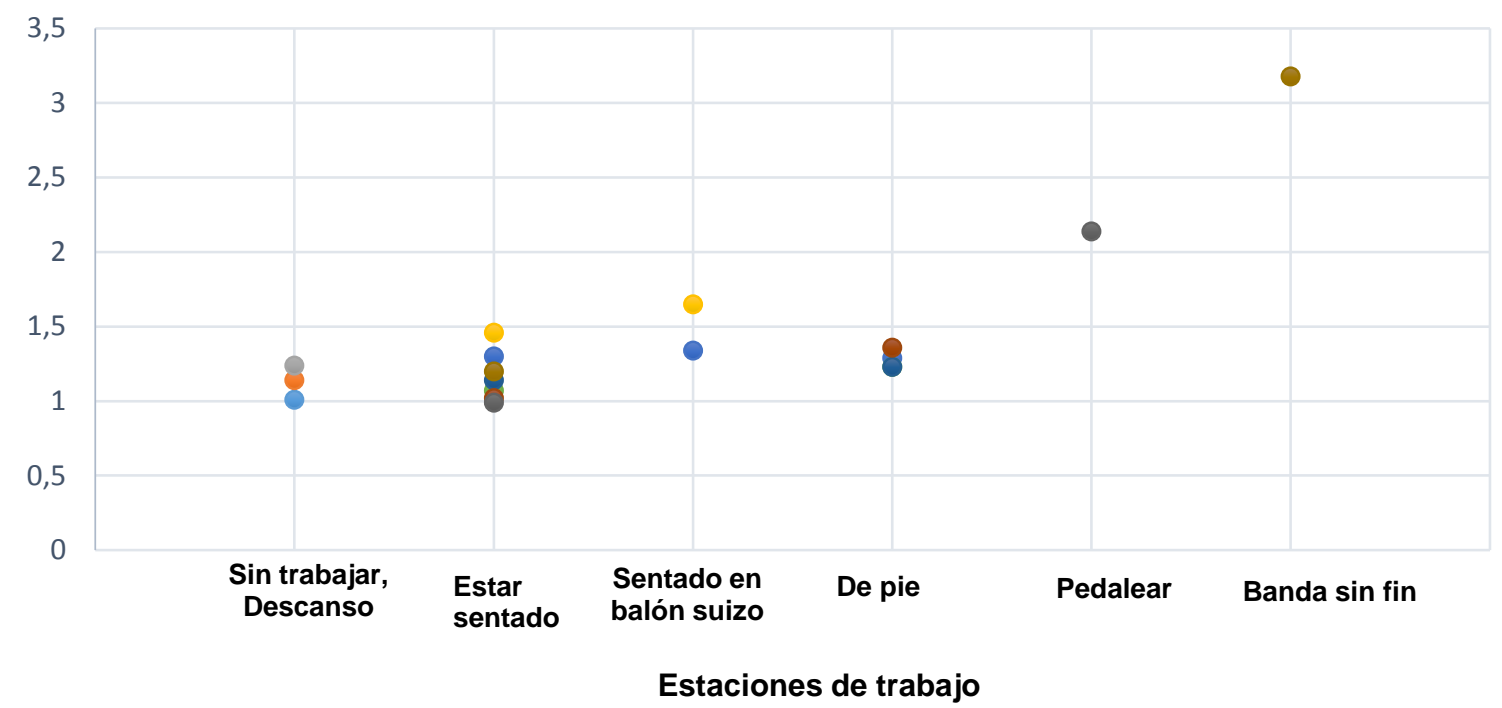

Figura 2. Resumen de los valores reportados en las publicaciones para la tasa promedio de gasto energético al utilizar estaciones de trabajo tradicionales y alternativas. Adaptado y actualizado de TudorLocke et al. (2014). Referencias: (A) Wright et al., 2015 [peso normal]; (B) Wright et al., 2015 [sobrepeso]; (C) Wright et al., 2015 [obesos]; (D) Dickin et al., 2015; (E) Speck et al., 2011; (F) Benden et al., 2014 [Otoño]; (G) Benden et al., 2014 [Primavera]; (H) Reiff et al., 2012; (I) Carr et al., 2014; (J) Levine et al., 2007.

A diferencia de las estaciones de trabajo reactivas, las estaciones alternativas proactivas, claramente, elevan las tasas de gasto energético. Carr et al. (2014) encontraron que el gasto energético se duplicó al pasar de estar simplemente sentados a pedalear mientras estaban sentados: los participantes pedalearon por 30 minutos y quemaron un promedio de $288.7 \pm$ 100.4 kJ (69 $\pm 24 \mathrm{kcal})$. Los datos de Levine \& Miller (2007) para una estación de trabajo con banda sin fin los llevaron a concluir que al hacer caminata a un poco más de $1.6 \mathrm{~km} / \mathrm{h}$ mientras

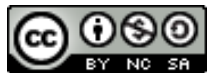

Esta obra está bajo una 
trabajaban, su gasto energético se elevó en 418 kJ/h (100 kcal/h), en comparación con estar sentados quietos. Las estaciones de trabajo activas les permiten a los trabajadores involucrarse en la actividad física a su propia intensidad o de manera intermitente. Cuanto más alta la intensidad y mayor la duración, tanto mayor será el gasto energético.

Respuestas cardiovasculares. El Sistema cardiovascular se ve afectado negativamente por la inactividad prolongada, la cual incluye el pasar sentado. La elevación aguda de la frecuencia cardíaca y la presión arterial mediante la actividad física es beneficiosa para fortalecer el corazón, aumentar el flujo de sangre y promover la dilatación de los vasos sanguíneos. Carr et al. (2014) sometieron a prueba los efectos fisiológicos de estar sentado, pero en forma activa, con un instrumento de pedales colocado bajo el escritorio. En ese estudio se encontró que la frecuencia cardíaca aumentó significativamente durante el tiempo sentados, mientras se llevaba a cabo un pedaleo proactivo $(89 \pm 11 \mathrm{lpm})$, en comparación con pasar sentados sin mucho movimiento $(75 \pm 12 \mathrm{lpm})$; la presión arterial sistólica también se elevó significativamente $(122 \pm 20$ vs. $109 \pm 11 \mathrm{mmHg})$. La gran pregunta es si las estaciones de trabajo activas ofrecen un estímulo lo suficientemente importante para lograr adaptaciones crónicas, a saber, una disminución en la frecuencia cardíaca en reposo y en la presión arterial a largo plazo; asimismo, nos referiremos a ella en la próxima sección.

También se ha identificado el pasar sentado ante un escritorio como un factor de riesgo de la trombosis venosa profunda (Dunstan, Howard, Healy, \& Owen, 2012a). Aunque comúnmente se ha considerado como una preocupación para la salud de los adultos mayores y aquellas personas que hacen viajes largos en avión, el estudio de caso de un varón de 32 años de edad, que pasaba demasiado tiempo en una estación de cómputo casi sin moverse, sacó a la luz el tema (Beasley, Raymond, Hill, Nowitz, \& Hughes,2003). Algunas investigaciones experimentales posteriores lograron identificar cambios hematopoyéticos, así como la expresión de genes del músculo esquelético que podrían contribuir al desarrollo de una trombosis, como resultado de la inactividad física provocada por pasar demasiado tiempo sentado o por la simulación de pasar demasiado tiempo en esta posición (Howard et al 2013; Zderic and Hamilton 2012). La interrupción del tiempo sentado ante la estación de trabajo, con períodos de 2 minutos de caminata a unos $3.2 \mathrm{~km} / \mathrm{h}$ tres veces por hora, logró reducir la aparición de factores sanguíneos que promueven la coagulación y el riesgo de trombosis (Howard et al. 2013). Un estudio de caso individual y los análisis de tejidos de animales mostraron que las sesiones posteriores de actividad física no revierten la supresión de la lípidofosfato-fosfatasa-1, sustancia que tendría un efecto protector al degradar los factores que promueven la trombosis (Zderic y Hamilton, 2012). Una vez más, esto sugiere que la actividad intermitente para interrumpir el tiempo prolongado sentado es importante, en este caso, para prevenir la hemostasis y los riesgos de trombosis.

Respuestas metabólicas. La actividad de bajo nivel y las interrupciones del tiempo que pasamos sentados en las estaciones de trabajo podrían ayudar a aumentar la tolerancia a la glucosa. Al reclutar las unidades motoras para la contracción muscular, aún a intensidad baja, 
es posible que se pueda mejorar la sensibilidad del tejido a la insulina; esto explicaría los reportes de mejorías agudas en la tolerancia a la glucosa (Dunstan et al., 2012b; Dempsey et al., 2016; Healy et al 2007). Aún está por verse si esto disminuye el riesgo de diabetes tipo 2.

Respuestas crónicas al uso de estaciones de trabajo alternativas. Las investigaciones prospectivas de largo plazo sobre los efectos de la utilización de estaciones de trabajo alternativas son limitadas. Entre los beneficios crónicos del aumento del NEAT o la actividad física están la pérdida de peso, mejorías en el sistema cardiovascular, menor presión arterial, disminución de los niveles de colesterol y una reducción del riesgo de enfermedades asociadas al estilo de vida sedentario. Se parte del supuesto que los trabajadores participarían en la actividad de su estación de trabajo durante la mayor parte del día o durante la jornada completa.

Pérdida de peso. La ganancia de peso se da con un equilibrio energético positivo, aun si es de solamente $418 \mathrm{~kJ}$ diarios (100 kcal diarias), siempre y cuando el desequilibrio se dé consistentemente (Hill, 2003). Según el Centro para el Control y la Prevención de las Enfermedades de los EE.UU., una cantidad moderada de actividad física equivale aproximadamente a $627 \mathrm{~kJ} / \mathrm{d}$ (150 kcal por día), o 4,180 kJ semanales (1,000 kcal por semana) (Health and Human Services, 2008). Además, se mencionó previamente que el gasto energético aumenta cuando se utilizan estaciones de trabajo alternativas, en comparación con el gasto obtenido en una estación de trabajo en la que se pasa sentado (Figura 2). Incluso la elevación mínima en el gasto energético, derivada del uso de algunas de las estaciones de trabajo alternativas estáticas, podría acumularse a lo largo de meses y años para contribuir a los beneficios del control del peso (Reiff et al., 2012; Dickin et al., 2015). A continuación, iniciamos con los resultados teóricos antes de analizar los resultados empíricos.

A partir del dato del gasto de sentarse en equilibrio sobre un balón suizo, el gasto adicional comparado con simplemente estar sentado quieto sería de unas $2.3 \mathrm{~kJ} / \mathrm{min}(0.55 \mathrm{kcal}$ por minuto); en una jornada de trabajo de 8 horas, la diferencia acumulada sería de $1088 \mathrm{~kJ}$ (260 kcal) (Faries, Bartholomew, \& McCallister, 2011). Este aumento les permitiría a los trabajadores un gasto adicional de 5,439 kJ (1,300 kcal) semanales. Dickin et al. (2015) sugirieron que, a pesar de tratarse de una elevación muy leve en el gasto calórico por usar el balón suizo como asiento en el trabajo, a lo largo de un año sería posible quemar entre 1.8 y $2.3 \mathrm{~kg}$ de grasa.

A partir de datos obtenidos en estudiantes de edad universitaria, Reiff et al. (2012) extrapolaron a jóvenes de edad colegial, especulando que estos estudiantes gastarían $477 \mathrm{~kJ}$ (114 kcal) adicionales por día de clases, o 85,609 kJ (20,461 kcal) anuales, usando como referencia el tiempo promedio que esos estudiantes pasan sentados en sus escritorios. Esto se podría traducir en una pérdida potencial de peso de unos $2.6 \mathrm{~kg}$ por año gracias a los escritorios para trabajar de pie. Estos efectos agudos relativamente despreciables de la utilización de estaciones de trabajo reactivas, podrían traducirse en beneficios crónicos para la 
salud en comparación con pasar sentado, siempre y cuando la conducta se dé en forma consistente.

Las estaciones de trabajo proactivas han mostrado tasas de gasto energético aún más altas, lo cual facilitaría la pérdida de peso o su control, siempre y cuando se utilicen en forma regular y los trabajadores no compensen ingiriendo más energía o volviéndose menos activos fuera del horario de trabajo. El estudio que hicieron Carr et al. (2014) con la estación de trabajo con pedales mostró un aumento de $285 \mathrm{~kJ}$ (68 kcal) de gasto por hora. Los autores creen que dicho efecto podría ser adecuado para disminuir el riesgo de diabetes, pues facilitaría la pérdida de peso y mejoraría la tolerancia a la glucosa y la resistencia a la insulina (Carr et al., 2014). De la misma forma, Levine et al. (2007) mostraron que es posible aumentar el gasto energético $498 \mathrm{~kJ}$ (119 kcal) por hora o 3,983 kJ (952 kcal) por jornada laboral, si se utiliza una banda sin fin para caminar frente al escritorio. Para personas con obesidad, caminar en una banda sin fin en el trabajo durante dos o tres horas diarias podría convertirse en una pérdida de peso de 20 a $30 \mathrm{~kg}$ por año, si los demás componentes del equilibrio energético se mantuvieran constantes (Levine et al., 2007).

Ahora bien, mientras por un lado es cierto que los datos empíricos muestran beneficios, también queda claro que las proyecciones han prometido demasiado. En el único estudio longitudinal, Koepp et al. (2013) llevaron a cabo una observación de empleados en trabajos sedentarios. A cada empleado se le instaló una banda sin fin con escritorio para que la usara por un año, con instrucciones de caminar en la banda mientras hacía todas las actividades normales del trabajo. Con esta intervención, el promedio de pérdida de peso fue de $1.4 \mathrm{~kg}$ para todo el grupo, $2.3 \mathrm{~kg}$ en promedio para los que eran obesos, luego de 12 meses. La circunferencia abdominal disminuyó significativamente en todos los participantes (de $95 \pm 19$ a

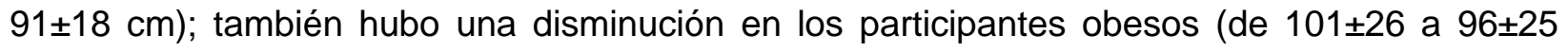
$\mathrm{cm}$ ). En este estudio no se incluyó un grupo control, pero entre los 23 participantes que se anotaron y esperaron 6 meses hasta que se inició la intervención del escritorio con banda sin fin, el peso corporal se mantuvo estable. A pesar de que el diseño de este estudio no utilizó los componentes clásicos de un experimento, los resultados sugieren que las estaciones de trabajo proactivas parecieran ofrecer mayores oportunidades para que los trabajadores se mantengan voluntariamente activos durante la jornada laboral y podrían promover la pérdida de peso, especialmente en las personas obesas.

Enfermedades cardiovasculares y metabólicas. El estudio publicado por Koepp et al. (2013) también analizó los efectos crónicos de la estación de trabajo con banda sin fin, sobre el sistema cardiovascular, la glucosa en sangre y los niveles de colesterol y triglicéridos antes, durante y después de la intervención de un año. Para el grupo completo $(n=36)$, la presión arterial sistólica en reposo disminuyó levemente, pero la reducción fue estadísticamente significativa ( $132 \pm 13$ vs. $129 \pm 13 \mathrm{mmHg}, \mathrm{p}<0.05)$. Además, el HDL aumentó desde los valores iniciales $(55 \pm 20$ vs. $59 \pm 23 \mathrm{mg} / \mathrm{dl}, \mathrm{p}<0.05)$. No se reportaron cambios estadísticamente significativos en la glicemia ni en los niveles de LDL, TSH ni triglicéridos. En términos generales, los cambios positivos en la presión arterial y el HDL son resultados prometedores 
para el mejoramiento de la salud de las personas, específicamente las personas obesas, con la esperanza de disminuir los riesgos de enfermedades cardiovasculares, síndrome metabólico y mortalidad.

Función cognitiva. Un objetivo crítico para cualquier estación de trabajo alternativa que promueva la actividad física, es que no distraiga a los trabajadores ni interfiera con su productividad. Tanto el trabajador como el patrono que invertiría en las estaciones de trabajo activas tienen un interés personal en este punto. Superficialmente, se podría asumir que las estaciones de trabajo reactivas, de baja intensidad, tienen una menor probabilidad de interferencia, mientras las estaciones proactivas podrían exigir atención mental para mantener el movimiento, lo cual desvía la concentración del tema del trabajo de escritorio. Las investigaciones no son claras a este respecto: alguna evidencia de hecho sugiere que las estaciones de trabajo alternativas, tanto las reactivas como las proactivas, podrían mejorar la productividad cognitiva, la percepción de la calidad del trabajo, la función cognitiva y el estado de ánimo, luego de que los trabajadores se cambian de una estación de trabajo sentado convencional (Dutta, Koepp, Stovitz, Levine, \& Pereira, 2014; Koepp et al., 2013; Larson et al., 2015; Mehta, Shortz, \& Benden 2015; Roemmich, 2014).

Mehta et al. (2015) estudiaron los beneficios neurológicos que experimentan los estudiantes al usar escritorios para trabajar de pie. Un hallazgo preliminar es que hubo entre 7 y $14 \%$ de mejoría en el rendimiento cognitivo en varias tareas de memoria (Mehta et al., 2015). También se reportó que los maestros percibieron que estos escritorios facilitaron el aprendizaje de los estudiantes sin causar distracción (Mehta et al., 2015). En otro estudio, los trabajadores que cambiaron un $50 \%$ del tiempo que pasaban sentados por trabajo de pie también aumentaron su sensación de bienestar y energía, disminuyeron la fatiga y no experimentaron impacto alguno en su productividad (Dutta et al., 2014). Al inicio de esta última intervención, los participantes sufrieron de dolor en las extremidades inferiores y en la espalda conforme pasaban más tiempo de pie. Sin embargo, para la segunda semana, estas molestias se resolvieron, de modo que los participantes estuvieron dispuestos a continuar utilizando la estación de trabajo ajustable, para trabajar sentados o de pie, aún después de la conclusión del estudio.

Varios investigadores han expresado su preocupación por el efecto negativo que pueden tener las estaciones de trabajo dinámicas sobre la productividad. Ciertamente, la preocupación por la seguridad de cada persona mientras se mueve en una banda sin fin y lleva a cabo trabajo intelectual relacionado con su ocupación es razonable, pero no está necesariamente justificada. Las estaciones de trabajo con pedales o banda sin fin se pueden ajustar a intensidades que son cómodas y manejables para los trabajadores. La duración de estas actividades podría también ser intermitente a lo largo del día, a saber, unos 10 minutos de pedaleo o caminata por cada hora de trabajo. Para respaldar esta estrategia, Elmer y Martin (2014) llevaron a cabo un experimento en el cual los participantes debían transcribir el Discurso de Gettysburg mientras estaban sentados quietos frente al escritorio o mientras pedaleaban frente a este. El estudio encontró que mientras los participantes pedaleaban a una potencia de 
$38 \mathrm{~W}$, el tiempo para cumplir la tarea de mecanografía ( $7.7 \pm 1.5 \mathrm{vs} .7 .6 \pm 1.6 \mathrm{~min})$ y el número de errores (3.3 \pm 4.6 vs. $3.8 \pm 2.7$ errores) no mostraron diferencias estadísticamente significativas con la ejecución de la tarea sentados quietos. Koepp et al. (2013) también concluyeron que, en su estudio de un año con la estación de trabajo con banda sin fin, el desempeño laboral no se vio afectado, según reportes de los supervisores y encuestas semanales. Por otra parte, Thompson y sus colegas (2011) mostraron una disminución en la productividad con las estaciones de trabajo con banda sin fin: después de recibir entrenamiento por 4 horas en el uso de las estaciones, se les pidió a los participantes que transcribieran grabaciones durante 8 horas, ya sea sentados quietos o caminando en la banda sin fin. La precisión no cambió, sin embargo, la velocidad fue un $16 \%$ más lenta con la banda sin fin en comparación con trabajar sentados. Los beneficios potenciales del trabajo caminando en una banda sin fin son inmensos, por lo cual en ese estudio se recomendó que debían ofrecerse más de 4 horas de entrenamiento para no perjudicar la productividad (Thompson, 2011).

\section{Conclusión}

Los efectos perjudiciales de pasar mucho tiempo sentado están bien establecidos; el trabajo de escritorio es claramente un componente importante del estilo de vida sedentario que contribuye a la obesidad y a otros riesgos de enfermedades crónicas. Es nuestra cauta conclusión, conforme a las investigaciones actuales, que las estaciones de trabajo alternativas podrían contrarrestar los efectos negativos de pasar largos períodos sentado frente a una estación de trabajo tradicional de escritorio. Para elevar la tasa metabólica, pareciera que las estaciones de trabajo activas y dinámicas, como caminar en una banda sin fin o pedalear, tienen un mayor impacto que las estaciones estáticas o reactivas como trabajar de pie o sentado en un balón suizo; sin embargo, los datos actualmente provienen de un número limitado de estudios. Por lo tanto, es necesario explorar algunas opciones adicionales. Las mejoras fisiológicas, tanto agudas como crónicas, relacionadas con las actividades de estaciones de trabajo alternativas, prometen beneficios para la salud, en particular si no disminuyen el desempeño cognitivo necesario para el trabajo. Actualmente, no está claro si los beneficios se derivan de las contracciones musculares 0 del gasto energético; independientemente de ello, no se deberían malinterpretar los beneficios de las estaciones de trabajo alternativas, de tal manera que se concluya que la actividad de muy baja intensidad puede sustituir al ejercicio planificado. Más bien, aquellas deberían ser una intervención adjunta en la gran variedad de estrategias para llevar un modo de vida que permita el control del peso corporal y la salud óptima.

\section{Agradecimientos}

Le damos las gracias a Laura Swan por su revisión diligente y por la retroalimentación brindada durante la preparación de este manuscrito. 
Traducción al español: Luis Fernando Aragón-Vargas, Ph.D., FACSM. Gracias al Lic. José Ángel Ramírez Obando por sus valiosos comentarios al borrador de la traducción.

\section{Referencias}

Ainsworth, B. E., Haskell, W. L., Whitt, M. C., Irwin, M. L., Swartz, A. M., Strath, S. J., ... Leon, A. S. (2000). Compendium of physical activities: An update of activity codes and MET intensities. Medicine and Science in Sports and Exercise, 32(9), 498-516. doi: http://dx.doi.org/10.1097/00005768-200009001-00009

Altieri, A., Tavani, A., Gallus, S., \& La vecchia, C. (2004). Occupational and leisure time physical activity and the risk of nonfatal acute myocardial infarction in Italy. Annals of Epidemiology, 14(7), 461-466. doi: http://dx.doi.org/10.1016/j.annepidem.2003.11.005

Beasley, R., Raymond, N., Hill, S., Nowitz, M., \& Hughes, R. (2003). eThrombosis: the 21st century variant of venous thromboembolism associated with immobility. European Respiratory Journal, 21(2), 374-376. doi: http://dx.doi.org/10.1183/09031936.03.00039403

Benden, M. E., Zhao, H., Jeffrey, C. E., Wendel, M. L., \& Blake, J. J. (2014). The evaluation of the impact of a stand-biased desk on energy expenditure and physical activity for elementary school students. International Journal of Environmental Research and Public Health, 11(9), 9361-9375. doi: http://doi.org/10.3390/ijerph110909361

Blaak, E.E., Westerterp, K.R., Bar-Or, O., Wouters, L.J.M., \& Saris, W.H.M. (1992). Total energy expenditure and spontaneous activity in relation to training in obese boys. American Journal of Clinical Nutrition, 55, 777-782. Retrieved from https://www.ncbi.nlm.nih.gov/pubmed/1550058

Carr, L. J., Maeda, H., Luther, B., Rider, P., Tucker, S. J., \& Leonhard, C. (2014). Acceptability and effects of a seated active workstation during sedentary work: A proof of concept study. International Journal of Workplace Health Management, 7(1), 2-15. doi: http://dx.doi.org/10.1108/IJWHM-03-2013-0008

Chu, A. H. Y., \& Moy, F. M. (2013). Associations of occupational, transportation, household and leisure-time physical activity patterns with metabolic risk factors among middle-aged adults in a middle-income country. Preventive Medicine, 57(Suppl), 14-17. doi: http://doi.org/10.1016/j.ypmed.2012.12.011

DeLany, J. P., Jakicic, J. M., Lowery, J. B., Hames, K. C., Kelley, D. E., \& Goodpaster, B. H. (2014). African American women exhibit similar adherence to intervention but lose less weight due to lower energy requirements. International Journal of Obesity, 38(9), 11471152. Retrieved from https://www.ncbi.nlm.nih.gov/pubmed/24352292

Dempsey, P. C., Larsen, R. N., Sethi, P., Sacre, J. W., Straznicky, N. E., Cohen, N. D., ... Dunstan, D. W. (2016). Benefits for Type 2 diabetes of interrupting prolonged sitting with brief bouts of light walking or simple resistance activities. Diabetes Research \& Care, 39(6), 964-972. doi: http://doi.org/10.2337/dc15-2336 
Dickin, D. C., Surowiec, R. K., \& Wang, H. (2015). Energy expenditure and muscular activation patterns through active sitting on compliant surfaces. Journal of Sport and Health Science. doi: http://doi.org/10.1016/j.jshs.2015.10.004

Drenowatz, C., Grieve, G. L., \& DeMello, M. M. (2015). Change in energy expenditure and physical activity in response to aerobic and resistance exercise programs. SpringerPlus, 4, 798. doi: http://doi.org/10.1186/s40064-015-1594-2

Dunstan, D. W., Howard, B., Healy, G. N., \& Owen, N. (2012a). Too much sitting- A health hazard. Diabetes Research and Clinical Practice, 97(3), 368-376. doi: http://doi.org/10.1016/j.diabres.2012.05.020

Dunstan, D. W., Kingwell, B. A., Larsen, R., Healy, G. N., Cerin, E., Hamilton, M. T., ... Owen, N. (2012b). Breaking up prolonged sitting reduces postprandial glucose and insulin responses. Diabetes Care, 35(5), 976-983. doi: http://doi.org/10.2337/dc11-1931

Dutta, N., Koepp, G. A., Stovitz, S. D., Levine, J. A., \& Pereira, M. A. (2014). Using sit-stand workstations to decrease sedentary time in office workers: A randomized crossover trial. International Journal of Environmental Research and Public Health, 11(7), 6653-6665. doi: http://doi.org/10.3390/ijerph110706653

Elmer, S. J., \& Martin, J. C. (2014). A cycling workstation to facilitate physical activity in office settings. Applied Ergonomics, 45(4), 1240-1246. doi: http://doi.org/10.1016/j.apergo.2014.03.001

Eriksen, D., Rosthøj, S., Burr, H., \& Holtermann, A. (2015). Sedentary work- Associations between five-year changes in occupational sitting time and body mass index. Preventive Medicine, 73, 1-5. doi: http://doi.org/10.1016/j.ypmed.2014.12.038

Faries, M. D. \& Bartholomew, J.B. \& McCallister, T. (2011). Acute effects of stability ball sitting on energy expenditure in the workplace. Medicine and Science in Sports and Exercise, 43(5). doi: http://dx.doi.org/10.1249/01.MSS.0000401664.38661.8e

Gibbs, B. B., Hergenroeder, A. L., Katzmarzyk, P. T., Lee, I.-M., \& Jakicic, J. M. (2015). Definition, measurement, and health risks associated with sedentary behavior. Medicine and Science in Sports and Exercise, 47(6), 1295-1300. doi: http://doi.org/10.1249/MSS.0000000000000517

Gimeno, D., Elovainio, M., Jokela, M., De Vogli, R., Marmot, M. G., \& Kivimaki, M. (2009). Association between passive jobs and low levels of leisure-time physical activity: The Whitehall II cohort study. Occupational and Environmental Medicine, 66(11), 772-776. doi: http://doi.org/10.1136/oem.2008.045104

Haskell, W. L., Lee, I.-M., Pate, R. R., Powell, K. E., Blair, S. N., Franklin, B. A., ... American Heart Association. (2007). Physical activity and public health: Updated recommendation for adults from the American College of Sports Medicine and the American Heart Association. Circulation, 116(9), 1081-1093. doi: http://doi.org/10.1161/CIRCULATIONAHA.107.185649

Health and Human Services. (2008). Physical activity guidelines for Americans: Be active, healthy, and happy. Retrieved from https://health.gov/paguidelines/pdf/paguide.pdf 
Healy, G. N., Dunstan, D. W., Salmon, J., Cerin, E., Shaw, J. E., Zimmet, P. Z., \& Owen, N. (2007). Objectively measured light-intensity physical activity is independently associated with 2-h plasma glucose. Diabetes Care, 30(6), 1384-1389. doi: http://doi.org/10.2337/dc07-0114

Hill, J. O., Melby, C., Johnson, S. L., \& Peters, J. C. (1995). Physical activity and energy requirements. The American Journal of Clinical Nutrition, 62(5), 1059-1066. Retrieved from http://ajcn.nutrition.org/content/62/5/1059S.abstract

Hill, J. O., Wyatt, H. R., Reed, G. W., \& Peters, J. C. (2003). Obesity and the environment: Where do we go from here? Science, 299(5608), 853-855. doi: http://doi.org/10.1126/science.1079857

Howard, B. J., Fraser, S. F., Sethi, P., Cerin, E., Hamilton, M. T., Owen, N., ... Kingwell, B. A. (2013). Impact on hemostatic parameters of interrupting sitting with intermittent activity. Medicine and Science in Sports and Exercise, 45(7), 1285-1291. doi: http://doi.org/10.1249/MSS.0b013e318285f57e

Hu, G., Qiao, Q., Silventoinen, K., Eriksson, J. G., Jousilahti, P., Lindström, J., ... Tuomilehto, J. (2003). Occupational, commuting, and leisure-time physical activity in relation to risk for Type 2 diabetes in middle-aged Finnish men and women. Diabetologia, 46(3), 322-329. Retrieved from http://link.springer.com/article/10.1007/s00125-003-1031-x

Johannsen, D. L., \& Ravussin, E. (2008). Spontaneous physical activity: Relationship between fidgeting and body weight control. Current Opinion in Endocrinology, Diabetes, and Obesity, 15(5), 409-415. doi: http://doi.org/10.1097/MED.0b013e32830b10bb

Katzmarzyk, P. T., Church, T. S., Craig, C. L., \& Bouchard, C. (2009). Sitting time and mortality from all causes, cardiovascular disease, and cancer. Medicine and Science in Sports and Exercise, 41(5), 998-1005. doi: http://doi.org/10.1249/MSS.0b013e3181930355

Koepp, G. A., Manohar, C. U., McCrady-Spitzer, S. K., Ben-Ner, A., Hamann, D. J., Runge, C. F., \& Levine, J. A. (2013). Treadmill desks: A 1-year prospective trial. Obesity, 21(4), 705-711. doi: http://doi.org/10.1002/oby.20121

Larson, M. J., LeCheminant, J. D., Carbine, K., Hill, K. R., Christenson, E., Masterson, T., \& LeCheminant, R. (2015). Slow walking on a treadmill desk does not negatively affect executive abilities: An examination of cognitive control, conflict adaptation, response inhibition, and post-error slowing. Frontiers in Psychology, 6 . doi: http://doi.org/10.3389/fpsyg.2015.00723

Lee, I.-M., Shiroma, E. J., Lobelo, F., Puska, P., Blair, S. N., Katzmarzyk, P. T., \& Lancet Physical Activity Series Working Group. (2012). Effect of physical inactivity on major non-communicable diseases worldwide: An analysis of burden of disease and life expectancy. The Lancet, 380(9838), 219-229. doi: http://doi.org/10.1016/S01406736(12)61031-9

Levi, F., Pasche, C., Lucchini, F., \& La Vecchia, C. (1999). Occupational and leisure time physical activity and the risk of breast cancer. European Journal of Cancer, 35(5), 775778. doi: http://doi.org/10.1016/S0959-8049(99)00051-9 
Levine, J. A. (2002). Non-exercise activity thermogenesis (NEAT). Best Practice \& Research. Clinical Endocrinology \& Metabolism, 16(4), 679-702. doi: http://dx.doi.org/10.1053/beem.2002.0227

Levine, J. A. (2005). Measurement of energy expenditure. Public Health Nutrition, 8(7), 11231132. doi: http://dx.doi.org/10.1079/phn2005800

Levine, J. A., \& Miller, J. M. (2007). The energy expenditure of using a "walk-and-work" desk for office workers with obesity. British Journal of Sports Medicine, 41(9), 558-561. doi: http://doi.org/10.1136/bjsm.2006.032755

Lin, T., Courtney, T. K., Lombardi, D. A., \& Verma, S. K. (2015). Association between sedentary work and BMI in a U.S. national longitudinal survey. American Journal of Preventive Medicine, 49(6), 117-123. doi: http://doi.org/10.1016/j.amepre.2015.07.024

Matthews, C. E., Chen, K. Y., Freedson, P. S., Buchowski, M. S., Beech, B. M., Pate, R. R., \& Troiano, R. P. (2008). Amount of time spent in sedentary behaviors in the United States, 2003-2004. American Journal of Epidemiology, 167(7), 875-881. doi: http://doi.org/10.1093/aje/kwm390

Matthews, C. E., George, S. M., Moore, S. C., Bowles, H. R., Blair, A., Park, Y., ... Schatzkin, A. (2012). Amount of time spent in sedentary behaviors and cause-specific mortality in US adults. The American Journal of Clinical Nutrition, 95(2), 437-445. doi: http://doi.org/10.3945/ajcn.111.019620

Mayo Clinic. (2014). Is sitting the new smoking? New science, old habit. Mayo Clinic Health Letter, 32(10), 4-5. Retrieved from https://www.ncbi.nlm.nih.gov/pubmed/26027033

Mehta, R. K., Shortz, A. E., \& Benden, M. E. (2015). Standing up for learning: A pilot investigation on the neurocognitive benefits of stand-biased school desks. International Journal of Environmental Research and Public Health, 13(1), 59.doi: http://doi.org/10.3390/ijerph13010059

Mummery, W. K., Schofield, G. M., Steele, R., Eakin, E. G., \& Brown, W. J. (2005). Occupational sitting time and overweight and obesity in Australian workers. American Journal of Preventive Medicine, 29(2), 91-97. doi: http://doi.org/10.1016/j.amepre.2005.04.003

Pate, R. R., Pratt, M., Blair, S. N., Haskell, W. L., Macera, C. A., Bouchard, C., ... King, A. C. (1995). Physical activity and public health. A recommendation from the Centers for Disease Control and Prevention and the American College of Sports Medicine. JAMA, 273(5), 402-407. doi: http://dx.doi.org/10.1001/jama.1995.03520290054029

Pate, R. R., O'Neill, J. R., \& Lobelo, F. (2008). The evolving definition of "sedentary." Exercise and Sport Sciences Reviews, 36(4), 173-178. doi: http://doi.org/10.1097/JES.0b013e3181877d1a

Racette, S. B., Schoeller, D. A., Kushner, R. F., \& Neil, K. M. (1995). Exercise enhances dietary compliance during moderate energy restriction in obese women. The American Journal of Clinical Nutrition, 62(2), 345-349. Retrieved from https://www.ncbi.nlm.nih.gov/pubmed/7625341 
Ravussin, E., Lillioja, S., Anderson, T. E., Christin, L., \& Bogardus, C. (1986). Determinants of 24-hour energy expenditure in man. Methods and results using a respiratory chamber. Journal of Clinical Investigation, 78(6), 1568-1578. http://dx.doi.org/10.1172/JCl112749

Redman, L. M., Heilbronn, L. K., Martin, C. K., de Jonge, L., Williamson, D. A., Delany, J. P., ... Pennington CALERIE Team. (2009). Metabolic and behavioral compensations in response to caloric restriction: Implications for the maintenance of weight loss. PloS One, 4(2). doi: http://doi.org/10.1371/journal.pone.0004377

Reiff, C., Marlatt, K., \& Dengel, D. R. (2012). Difference in caloric expenditure in sitting versus standing desks. Journal of Physical Activity \& Health, 9(7), 1009-1011. doi: http://dx.doi.org/10.1123/jpah.9.7.1009

Roemmich, J. (2014). Effect of height adjustable desks on standing time, discomfort, and productivity of office workers with sedentary occupations (37.8). The FASEB Journal, 28(1). Retrieved from http://www.fasebj.org/content/28/1_Supplement/37.8

Ryde, G. C., Brown, H. E., Gilson, N. D., \& Brown, W. J. (2014). Are we chained to our desks? Describing desk-based sitting using a novel measure of occupational sitting. Journal of Physical Activity \& Health, 11(7), 1318-1323. doi: http://doi.org/10.1123/jpah.2012-0480

Sedentary Behaviour Research Network, null. (2012). Letter to the editor: standardized use of the terms "sedentary" and "sedentary behaviours." Applied Physiology, Nutrition, and Metabolism = Physiologie Appliquée, Nutrition Et Métabolisme, 37(3), 540-542. doi: http://doi.org/10.1139/h2012-024

Speck, R.M., Schmitz, K.H. (2011). Energy expenditure comparison: A pilot study of standing instead of sitting at work for obesity prevention. Prevention Medicine, 52(3-4), 283-284. doi: http://dx.doi.org/10.1016/j.ypmed.2011.02.002

Tavani, A., Braga, C., Vecchia, C. L., Conti, E., Filiberti, R., Montella, M., ... Franceschi, S. (1999). Physical activity and risk of cancers of the colon and rectum: An Italian casecontrol study. British Journal of Cancer, 79, 1912-1916. doi: http://doi.org/10.1038/sj.bjc.6690304

Thivel, D., Chaput, J. P., Adamo, K. B., \& Goldfield, G. S. (2014). Is energy intake altered by a 10 -week aerobic exercise intervention in obese adolescents?. Physiology \& Behavior, 135, 130-134. Retrived from https://www.ncbi.nlm.nih.gov/pubmed/24952265

Thompson, W. G., \& Levine, J. A. (2011). Productivity of transcriptionists using a treadmill desk. Work, 40(4), 473-477. Retrieved from https://www.ncbi.nlm.nih.gov/pubmed/22130064

Thorp, A. A., Healy, G. N., Winkler, E., Clark, B. K., Gardiner, P. A., Owen, N., \& Dunstan, D. W. (2012). Prolonged sedentary time and physical activity in workplace and non-work contexts: a cross-sectional study of office, customer service and call centre employees. The International Journal of Behavioral Nutrition and Physical Activity, 9, 128. doi: http://doi.org/10.1186/1479-5868-9-128

Thune, I., Brenn, T., Lund, E., \& Gaard, M. (1997). Physical activity and the risk of breast cancer. The New England Journal of Medicine, 336(18), 1269-1275. http://doi.org/10.1056/NEJM199705013361801 
Tudor-Locke, C., Schuna, J. M., Frensham, L. J., \& Proenca, M. (2014). Changing the way we work: Elevating energy expenditure with workstation alternatives. International Journal of Obesity, 38(6), 755-765. Retrieved from https://www.ncbi.nlm.nih.gov/pubmed/24285335

Van der Ploeg, H. P., Chey, T., Korda, R. J., Banks, E., \& Bauman, A. (2012). Sitting time and all-cause mortality risk in 222497 Australian adults. Archives of Internal Medicine, 172(6), 494-500. doi: http://doi.org/10.1001/archinternmed.2011.2174

Warren, T. Y., Barry, V., Hooker, S. P., Sui, X., Church, T. S., \& Blair, S. N. (2010). Sedentary behaviors increase risk of cardiovascular disease mortality in men. Medicine and Science in Sports and Exercise, 42(5), 879-885. doi: http://doi.org/10.1249/MSS.0b013e3181c3aa7e

Willis, E. A., Herrmann, S. D., Honas, J. J., Lee, J., Donnelly, J. E., \& Washburn, R. A. (2014). Nonexercise energy expenditure and physical activity in the Midwest exercise trial 2. Medicine and Science in Sports and Exercise, 46(12), 2286-2294. doi: http://doi.org/10.1249/MSS.0000000000000354

Wright, T. G., Dawson, B., Jalleh, G., \& Guelfi, K. J. (2015). Influence of hormonal profile on resting metabolic rate in normal, overweight and obese individuals. Annals of Nutrition \& Metabolism, 66(2-3), 162-167. doi: http://doi.org/10.1159/000382080

Zderic, T. W., \& Hamilton, M. T. (2012). Identification of hemostatic genes expressed in human and rat leg muscles and a novel gene (LPP1/PAP2A) suppressed during prolonged physical inactivity (sitting). Lipids in Health and Disease, 11, 137. doi: http://doi.org/10.1186/1476-511X-11-137

Participación: A- Financiamiento, B- Diseño del estudio, C- Recolección de datos, D- Análisis estadístico e interpretación de resultados, E- Preparación del manuscrito. 